\title{
ESDA2012-82213
}

\section{OPTIMALITY CRITERIA FOR MEASUREMENT POSES SELECTION IN CALIBRATION OF ROBOT STIFFNESS PARAMETERS}

\author{
Yier Wu ${ }^{a, b}$, Alexandr Klimchik ${ }^{a, b}$, Anatol Pashkevich ${ }^{a, b}$, Stéphane Caro ${ }^{b}$, Benoît Furet ${ }^{b}$ \\ ${ }^{a}$ Ecole des Mines de Nantes, \\ 4 rue Alfred-Kastler, 44307 Nantes, France \\ ${ }^{b}$ Institut de Recherche en Communications et Cybernétique de Nantes, \\ UMR CNRS n ${ }^{\circ} 6597,1$ rue de la Noë, 44321 Nantes, France \\ Email: yier.wu@mines-nantes.fr
}

\begin{abstract}
The paper focuses on the accuracy improvement of industrial robots by means of elasto-static parameters calibration. It proposes a new optimality criterion for measurement pose selection in calibration of robot stiffness parameters. This criterion is based on the concept of the manipulator test pose that is defined by the user via the joint angles and the external force. The proposed approach essentially differs from the traditional ones and ensures the best compliance error compensation for the test configuration. The advantages of this approach and its suitability for practical applications are illustrated by numerical examples, which deal with calibration of elasto-static parameters of planar manipulator with rigid links and compliant actuated joints.
\end{abstract}

Keywords: robot calibration, stiffness parameters identification, selection of measurement poses, optimality criteria

\section{INTRODUCTION}

The aerospace and ship building industries intend to progressively replace conventional materials by composite ones that provide essential advantages from mechanical point of view, but at the same time introduce additional complexity in machining process. Conventional CNC-machines provide high stiffness and high accuracy, but the workspace is very limited. Besides, it is difficult to process the workpieces with complex shapes. These limitations are critical for the considered application. In this case, it is reasonable to consider robotic-based machining that may ensure large workspace and processing of complex shapes. However, machining of high performance materials with robot may cause essential compliance errors that influence on the quality of the final product. For this reason, in the design of roboticbased machining, stiffness modeling of robotic manipulators under external forces becomes a crucial issue.

Generally, the compliance errors depend on both applied loading and robot stiffness properties. Moreover, similar to geometrical errors they highly rely on the manipulator configuration and essentially differ throughout the workspace. So, in order to achieve maximal efficient compensation, it is required a reliable stiffness model, which takes into account both stiffness properties of all joints/links and correct values of all joint angles. One way to solve this problem is to improve the stiffness model by means of elasto-static calibration [1] that allows identifying the stiffness parameters from the measured robot end-effector positions.

The problem of robot calibration is in the focus of research community for many years [2]. However, most of the efforts have been made for kinematic calibration [3-6], only few works directly address the issue of elasto-static calibration $[7,8]$ and its influences on robot accuracy. Only very limited number of works 
address the issue of optimal measurement pose selection [9-11]. Despite the obvious fact that the calibration accuracy may be improved by increasing the number of experiments, the measurement poses may also affect the robot calibration [12]. It has been shown that the latter may significantly improve the identification accuracy [13]. This problem can be treated as determining a set of optimal measurement poses within the reachable joint space so that the affect of measurement errors on the identification of robot parameters is minimized.

In the experiment design theory, which can be obviously applied here, one of the key issues is comparison of the experimental plans. In order to find the optimal experimental plan, numerous quantitative performance measures have been proposed. They have different affects on the identification accuracy, and have been defined as the objectives of the optimization problems, associated with sets of measurement poses. In robotics, there exist two main trends in defining the objectives: one is the conventional optimality criteria [14-16], which operate with the trace/determinant of the covariance matrix. Another one is the observability indices [17-20], which are based on singular value decomposition of the Jacobian. Both trends have limitations that affects the calibration accuracy in different manner. Besides, the developed performance measures that operate with abstract notions, and as a result they are not in a good agreement with the industrial requirements. This motivates a research direction of this work.

In this paper, to evaluate the quality of measurement poses used in calibration experiments, a new criterion that is based on the concept of manipulator test-pose is introduced. In contrast to the existing criteria, the proposed one has a clear physical meaning directly related to the robot accuracy, and allows essentially improving the accuracy of compliance errors compensation via proper selection of the measurement poses. The main advantages and practical significance of the proposed criteria are illustrated with the elasto-static calibration of 2-link planar manipulator. For the presented example, a series of optimal pose selection maps with respect to different link length ratio are presented.

\section{PROBLEM STATEMENT}

In order to address the problem of elasto-static calibration, let us define the required mathematic models: geometric and elasto-static ones. The geometric model that defines the robot end-effector position as a function of the joint angles and link lengths can be expressed as

$$
\mathbf{p}=g(\mathbf{q}, \mathbf{L})
$$

where $\mathbf{p}$ is the end-effector location (position and orientation), vector $\mathbf{q}$ is the joint angles and vector $\mathbf{L}$ collects the link length.

In accordance with [21], the displacement of the end- effector under the external loading of serial manipulator is

$$
\Delta \mathbf{p}=\mathbf{J} \mathbf{k}_{\theta} \mathbf{J}^{T} \mathbf{F}
$$

where the Jacobian matrix $\mathbf{J}$ can be computed via differentiation of the geometric model (1); $\Delta \mathbf{p}$ is the robot end-effector displacement caused by external loading; $\mathbf{k}_{\theta}$ is a matrix that aggregates compliances $\left\{k_{1}, \cdots, k_{n}\right\}$ of the actuated joints; $\mathbf{F}$ is the external force.

It is assumed that all the geometric parameters $\{\mathbf{q}, \mathbf{L}\}$ of model (1) are well calibrated. So, for the unloaded mode $(\mathbf{F}=0)$, the vector $\mathbf{q}$ is equal to the nominal value of the joint angles $\mathbf{q}^{0}$. Because of the loading $\mathbf{F} \neq 0$, the joint angles include deflections, i.e. $\mathbf{q}=\mathbf{q}^{0}+\delta \mathbf{q}$, where $\delta \mathbf{q}$ is the vector of joint displacements under the external loading $\mathbf{F}$. Thus, in order to compensate the undesired displacements caused by $\mathbf{F}$, the elasto-static parameters $\left\{k_{1}, \cdots, k_{n}\right\}$ must be identified precisely. For this purpose, it is proposed to use calibration technique.

For elasto-static model, each calibration experiment produces three vectors $\left\{\Delta \mathbf{p}_{i}, \mathbf{q}_{i}, \mathbf{F}_{i}\right\}$, which define the displacements of robot end-effector, the corresponding joint angles and the external forces respectively, where $i$ is the experiment number. Correspondingly, the calibration procedure may be treated as the best fitting of the experimental data $\left\{\Delta \mathbf{p}_{i}, \mathbf{q}_{i}, \mathbf{F}_{i}\right\}$ by using the stiffness model (2) that can be solved using the standard least-square technique.

However in practice, the calibration includes measurement of Cartesian coordinates that accommodate errors $\varepsilon$, which are assumed to be i.i.d (independent identically distributed) random values with zero expectation and standard deviation $\sigma$. The errors for the joint variables are assumed to be relatively small. Because of the errors in the measurements, the desired values $\left\{k_{1}, \cdots, k_{n}\right\}$ are always identified approximately. So, the problem of interest is to evaluate the identification accuracy for the identified parameters and to propose a technique for selecting the set of joint variables $\mathbf{q}_{i}$ and external forces $\mathbf{F}_{i}$ that leads to accuracy improvement.

Usually, the optimality criteria that evaluate the quality of calibration plans are based on the covariance matrix of the identified parameters. In this particular case, the parameters of $\mathbf{k}_{\theta}$ have different influences on the end-effector displacements, moreover, their influence varies throughout the workspace. To overcome this difficulty, in this work it is assumed that the "calibration quality" is evaluated for the so-called test configuration $\left\{\mathbf{q}^{*}, \mathbf{F}^{*}\right\}$, which is given by the user and for which it is required to have the best positioning accuracy under the influence of external loading. So, original calibration problem should be solved with respect to the given constraint.

To solve this general problem, two subproblems should be 
considered: (i) to propose an optimality criterion that is adapted to the elasto-static parameters calibration of industrial manipulator; (ii) to find optimal configuration of robot calibration for given test one that provide the highest position accuracy.

\section{EVALUATION OF IDENTIFICATION ACCURACY Influence of Measurement Errors}

For computational convenience, the linear relation (2) where the desired parameters $\left\{k_{1}, \cdots, k_{n}\right\}$ are arranged in the compliance matrix $\mathbf{k}_{\theta}$ of size $n \times n$ should be rewritten into the following form

$$
\Delta \mathbf{p}_{i}=\mathbf{A}_{i} \mathbf{k}
$$

where the $n \times 1$ vector $\mathbf{k}$ collects the joint compliances $\left\{k_{1}, \cdots, k_{n}\right\}$. Matrix $\mathbf{A}_{i}$ is defined by the columns of Jacobian and the external force and can be expressed as

$$
\mathbf{A}_{i}=\left[\mathbf{J}_{i}^{1} \mathbf{J}_{i}^{1 T} \mathbf{F}_{i}, \cdots, \mathbf{J}_{i}^{n} \mathbf{J}_{i}^{n T} \mathbf{F}_{i}\right] \quad(i=\overline{1, m})
$$

where $\mathbf{J}_{i}^{n}$ is the $n^{\text {th }}$ column vector of Jacobian matrix for the $i^{\text {th }}$ experiment, $m$ being the number of experiments. From the identification theory, the joint compliances can be obtained from Eq. (3) using least square method, which minimizes the residuals for all experimental data. So, the corresponding optimization problem can be formulated as

$$
\sum_{i=1}^{m}\left(\mathbf{A}_{i} \mathbf{k}-\Delta \mathbf{p}_{i}\right)^{T}\left(\mathbf{A}_{i} \mathbf{k}-\Delta \mathbf{p}_{i}\right) \rightarrow \min _{\mathbf{q}_{i}, \mathbf{F}_{i}}
$$

The solution of this optimization problem provides the estimation of desired parameters, which can be computed as

$$
\mathbf{k}_{0}=\left(\sum_{i=1}^{m} \mathbf{A}_{i}^{T} \mathbf{A}_{i}\right)^{-1}\left(\sum_{i=1}^{m} \mathbf{A}_{i}^{T} \Delta \mathbf{p}_{i}\right)
$$

Taking into account that the measurement errors exist in the calibration experiments, Eq. (3) should be rewritten in the following form

$$
\Delta \mathbf{p}_{i}=\mathbf{A}_{i} \mathbf{k}+\varepsilon_{i}
$$

where $\varepsilon_{i}$ is the measurement errors in the $i^{\text {th }}$ experiment with expectation $\mathrm{E}\left(\varepsilon_{i}\right)=0$ and variance $\mathrm{E}\left(\varepsilon_{i}^{T} \varepsilon_{i}\right)=\sigma^{2}$. It is evident that the measurement errors have affect on the identification accuracy of $\mathbf{k}$. So, the estimation of the desired parameters $\mathbf{k}$ has the form

$$
\mathbf{k}=\left(\sum_{i=1}^{m} \mathbf{A}_{i}^{T} \mathbf{A}_{i}\right)^{-1}\left(\sum_{i=1}^{m} \mathbf{A}_{i}^{T}\left(\Delta \mathbf{p}_{i}-\varepsilon_{i}\right)\right)
$$

As follows from Eq. (8), the latter expression produces unbiased estimates

$$
\mathrm{E}(\mathbf{k})=\mathbf{k}_{0}
$$

where $\mathrm{E}(\mathbf{k})$ is the expectation of the identified parameters, which is assumed to be equal to the real value of $\mathbf{k}_{0}$. It can be proved that the covariance matrix of compliance parameters [22], defining the identification accuracy, can be expressed as

$$
\operatorname{cov}(\mathbf{k})=\left(\sum_{i=1}^{m} \mathbf{A}_{i}^{T} \mathbf{A}_{i}\right)^{-1} \mathrm{E}\left(\sum_{i=1}^{m} \mathbf{A}_{i}^{T} \varepsilon_{i}^{T} \varepsilon_{i} \mathbf{A}_{i}\right)\left(\sum_{i=1}^{m} \mathbf{A}_{i}^{T} \mathbf{A}_{i}\right)^{-1}
$$

Then, taking into account that $\mathrm{E}\left(\sum_{i=1}^{m} \varepsilon_{i}^{T} \varepsilon_{i}\right)=\sigma^{2} \mathbf{I}$, where $\mathbf{I}$ is the $n \times n$ identity matrix, Eq. (10) can be simplified to

$$
\operatorname{cov}(\mathbf{k})=\sigma^{2}\left(\sum_{i=1}^{m} \mathbf{A}_{i}^{T} \mathbf{A}_{i}\right)^{-1}
$$

where $\sigma$ is the standard deviation of the measurement errors. So, for the considered problem, the impact of the measurement errors is defined by the matrix sum $\sum_{i=1}^{m} \mathbf{A}_{i}^{T} \mathbf{A}_{i}$ that is also called the information matrix.

Obviously, in order to have the smallest dispersion of identification errors, the elements of covariance matrix should be as small as possible. However, this is a multi-objective optimization problem, which means that minimization of one element may possibly increase others. For this reason, let us concentrate on the analysis of the existing optimality criteria and their applicability to the considered application in the next section.

\section{Existing Optimality Criteria}

In classical regression analysis, there are several conventional optimality criteria that operate with the trace and/or determinant of covariance or information matrices. The most commonly used among them are based on A-, T-, D-, G-optimality principles and maximal diagonal covariance (MDC). In addition, in robot calibration, there exist many observability indices based on the singular value decomposition of the Jacobian. For the considered application, they can be obtained from SVD of matrix A, which contains the Jacobian and the external force. More details concerning these criteria are presented in Table 1.

It should be mentioned that all of the optimization criteria, which are presented in Table 1 solve the minimization problem (11) in different manners. As a result, they may provide different optimal solutions. So, in order to have good accuracy of robot parameters, it is important to select a proper objective. The main disadvantage of these approaches is that the objectives are not directly related to the robot accuracy, which is a critical issue in the industry. Even if they may increase the identifica- 
Table 1. Optimization functions for existing optimality criteria

\begin{tabular}{ll}
\hline \hline Optimality Criterion & Optimization Function \\
\hline A-optimality & $\operatorname{trace}\left(\left(\mathbf{A}^{T} \mathbf{A}\right)^{-1}\right) \rightarrow \min _{\mathbf{q}, \mathbf{F}}$ \\
T-optimality & $\operatorname{trace}\left(\mathbf{A}^{T} \mathbf{A}\right) \rightarrow \max _{\mathbf{q}, \mathbf{F}}$ \\
D-optimality & $\operatorname{det}\left(\mathbf{A}^{T} \mathbf{A}\right) \rightarrow \max _{\mathbf{q}, \mathbf{F}}$ \\
G-optimality & $\max \{d[\mathbf{H}]\} \rightarrow \min _{\mathbf{q}, \mathbf{F}}$ \\
MDC & $\max \left\{d\left[\left(\mathbf{A}^{T} \mathbf{A}\right)^{-1}\right]\right\} \rightarrow \min _{\mathbf{q}, \mathbf{F}}$ \\
Product of singular values $\left(O_{1}\right)$ & $\frac{s \sqrt{\sigma_{s} \cdots \sigma_{1}}}{\sqrt{m}} \rightarrow \max _{\mathbf{q}, \mathbf{F}}$ \\
Inverse condition number $\left(O_{2}\right)$ & $\frac{\sigma_{s}^{2}}{\sigma_{1}{ }^{3}} \rightarrow \max _{\mathbf{q}, \mathbf{F}}$ \\
Minimum singular value $\left(O_{3}\right)$ & $\sigma_{s} \rightarrow \max _{\mathbf{q}, \mathbf{F}}$ \\
Noise amplification index $\left(O_{4}\right)$ & $\frac{\sigma_{s}^{2}}{\sigma_{1}} \rightarrow \max _{\mathbf{q}, \mathbf{F}}$ \\
\hline \hline 1 & $d[\cdot]$ extract diagonal elements of the matrix; \\
$2 \sigma_{s}$ minimum singular value; & \\
${ }^{3} \sigma_{1}$ maximum singular value; &
\end{tabular}

tion accuracy of the robot parameters, they can not provide the best prediction of the robot end-effector location. Therefore, a new optimality criterion that ensures the best robot end-effector positioning accuracy under external loading is required.

\section{Test-pose Based Approach}

In order to give a clear physical meaning that is related to the robot accuracy, a new optimality criterion is proposed. This criterion is based on the mean squared error of end-effector displacements and evaluates the ability to compensate the compliance errors for given test pose. It should be mentioned that similar approach has been used in prediction theory, but has never been applied in robot calibration [23].

Assuming that the measurement errors affect the identification accuracy, Eq. (7) can be expressed in a different manner:

$$
\Delta \mathbf{p}+\delta \mathbf{p}=\mathbf{A}_{t}(\mathbf{k}+\delta \mathbf{k})
$$

where $\delta \mathbf{p}$ stands for the deflection error, and $\delta \mathbf{k}$ describes the compliance error. $\mathbf{A}_{t}$ is defined by the given test pose using Eq. (3). Accordingly, Eq. (12) is equivalent to

$$
\delta \mathbf{p}=\mathbf{A}_{t} \delta \mathbf{k}
$$

So, the mean squared error of the joint compliances under the external force, which is defined using term $O_{t}$, can be expressed as

$$
O_{t}=\mathrm{E}\left(\delta \mathbf{p}^{T} \delta \mathbf{p}\right)
$$

In order to simplify Eq. (14), the term $\delta \mathbf{p}^{T} \delta \mathbf{p}$ could be replaced by $\operatorname{trace}\left(\delta \mathbf{p} \delta \mathbf{p}^{T}\right)$, such that $O_{t}$ has the following form

$$
O_{t}=\operatorname{trace}\left(\mathrm{E}\left(\mathbf{A}_{t} \delta \mathbf{k} \delta \mathbf{k}^{T} \mathbf{A}_{t}^{T}\right)\right)
$$

And taking into account that $\mathrm{E}\left(\delta \mathbf{k} \delta \mathbf{k}^{T}\right)$ is the covariance matrix of the desired parameters $\mathbf{k}$, using Eq. (11), $O_{t}$ can be reduced to

$$
O_{t}=\sigma^{2} \operatorname{trace}\left(\mathbf{A}_{t}\left(\sum_{i=1}^{m} \mathbf{A}_{i}^{T} \mathbf{A}_{i}\right)^{-1} \mathbf{A}_{t}^{T}\right)
$$

Analysis of Eq. (16) allows to formulate several remarks:

Remark 1 This approach is an extension of the conventional Aoptimality concept. It can be treated as the weighted trace of the covariance matrix of desired parameters, where the weighting coefficients are derived using the test pose. It also ensures low values of the covariance matrix elements and allows to combine multiple objectives with different units in a single scalar criterion.

Remark 2 From computational point of view, carrying out a single calibration experiment makes sense only when the number of parameters to be measured (the end-effector position for instance) is more than or equal to the number of parameters to be identified (the joint compliances). Otherwise, the system of equations is underdetermined. Besides, from classical regression analysis, it is known that the increasing of number of experiments also improves the identification accuracy. It can be proved that the proposed criterion is well adapted to both cases.

Remark 3 If the test pose and measurement poses are the same, which means that $\mathbf{A}_{i}=\mathbf{A}_{t}$, the expression for $O_{t}$ can be simplified to

$$
\left.O_{t}^{*}\right|_{\mathbf{A}_{i}=\mathbf{A}_{t}, i=\overline{1, m}}=\frac{n \sigma^{2}}{m}
$$

where $n$ is the number of identifiable parameters and $m$ is the number of measurement poses.

To demonstrate the advantages of the proposed criterion, some illustrative examples with comparison studies are in the consideration of the next section.

\section{ILLUSTRATIVE EXAMPLE}

\section{Geometric and Stiffness Models}

Let us consider a 2-link manipulator with rigid links and two compliant actuated joints located on the base and between the 


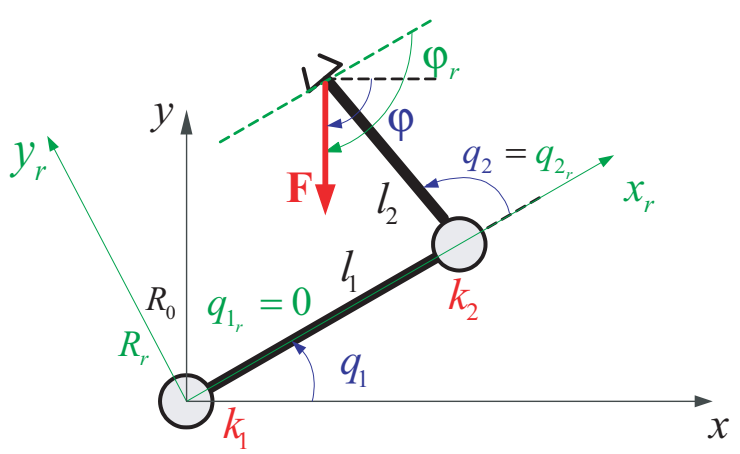

Figure 1. Geometry and statics of 2-link manipulator

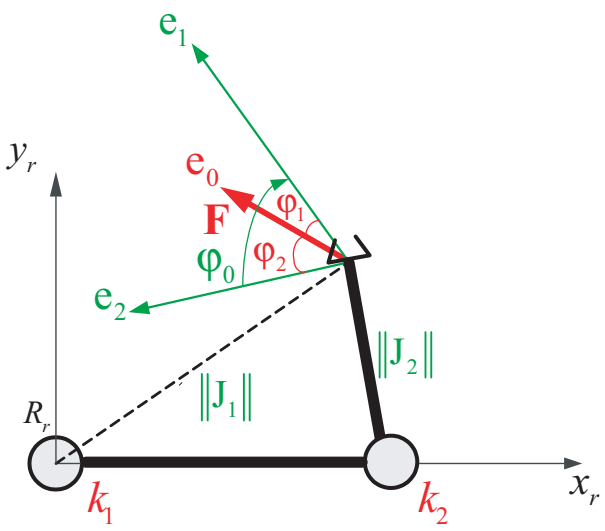

Figure 2. Geometry and statics of 2-link manipulator in vector representation

two links (Fig. 1). Its geometric model can be expressed as

$$
\left\{\begin{array}{l}
x=l_{1} \cos \left(q_{1}\right)+l_{2} \cos \left(q_{1}+q_{2}\right) \\
y=l_{1} \sin \left(q_{1}\right)+l_{2} \sin \left(q_{1}+q_{2}\right)
\end{array}\right.
$$

where $x$ and $y$ are Cartesian coordinates of the robot end-effector position to be measured, $q_{1}$ and $q_{2}$ are the joint angles that are the design variables, $l_{1}$ and $l_{2}$ are the link lengths. Assuming that the external force $\mathbf{F}$ has a fixed magnitude $F$, one more design variable $\varphi$ that defines the force direction should be introduced. As a result, the external force can be expressed as $\mathbf{F}=F[\cos (\varphi), \sin (\varphi)]^{T}$.

In order to obtain analytical solutions of optimal measurement poses for existing optimality criteria, it is reasonable to rewrite the geometric model of the considered manipulator in the local coordinate system $R_{r}$. In this coordinate system, the x-axis is coincided with the first link. And the joint angles and force direction can be defined in local frame $R_{r}$ using $q_{r 1}, q_{r 2}$ and $\varphi_{r}$ that have the following relations with $q_{1}, q_{2}$ and $\varphi$, respectively:

$$
\left\{q_{r 1}=0, \quad q_{r 2}=q_{2}, \quad \varphi_{r}=\varphi-q_{1}\right\}
$$

This allows us to reduce the number of design variables from three $\left\{q_{r 1}, q_{r 2}, \varphi_{r}\right\}$ to two $\left\{q_{r 2}, \varphi_{r}\right\}$. Accordingly, the Jacobian in local frame $R_{r}$ can be expressed as

$$
\mathbf{J}=\left[\begin{array}{r:c}
-l_{2} \sin \left(q_{2}\right) & -l_{2} \sin \left(q_{2}\right) \\
l_{1}+l_{2} \cos \left(q_{2}\right) & l_{2} \cos \left(q_{2}\right)
\end{array}\right]
$$

Using this expression, the stiffness model (2) can be simplified by rewriting the Jacobian matrix in vector form,

$$
\mathbf{J}=\left[\left\|\mathbf{J}_{1}\right\| \mathbf{e}_{1} \vdots\left\|\mathbf{J}_{2}\right\| \mathbf{e}_{2}\right]
$$

where the scalars $\left\|\mathbf{J}_{i}\right\|(i=1,2)$ refer to the Euclid norm of vector $\mathbf{J}_{i}$, and $\mathbf{e}_{i}$ defines the vector direction. It can be easily proved that, for the considered manipulator, $\left\|\mathbf{J}_{1}\right\|$ and $\left\|\mathbf{J}_{2}\right\|$ have the form

$$
\begin{aligned}
& \left\|\mathbf{J}_{1}\right\|=\sqrt{l_{1}{ }^{2}+l_{2}{ }^{2}+2 l_{1} l_{2} \cos \left(q_{2}\right)} \\
& \left\|\mathbf{J}_{2}\right\|=l_{2}
\end{aligned}
$$

Following the same principle, the scalar product $\mathbf{J}_{i}^{T} \mathbf{F}$ can be expressed as

$$
\mathbf{J}_{i}^{T} \mathbf{F}=\left\|\mathbf{J}_{i}\right\|\|\mathbf{F}\| \cos \left(\varphi_{i}\right)
$$

where $\|\mathbf{F}\|=F, \varphi_{i}$ stands for the angle between force direction $\mathbf{e}_{0}$ and direction of Jacobian column vector $\mathbf{e}_{i}$ (Fig. 2). It is also reasonable to introduce $\varphi_{0}$ that refers to the angle between two Jacobian column vectors, it has the relation of $\varphi_{0}=\varphi_{1}+\varphi_{2}$. In the frame of introduced notations, $\mathbf{A}$ should take the form

$$
\mathbf{A}=F\left[\left\|\mathbf{J}_{1}\right\|^{2} \cos \left(\varphi_{1}\right) \mathbf{e}_{1} \vdots\left\|\mathbf{J}_{2}\right\|^{2} \cos \left(\varphi_{2}\right) \mathbf{e}_{2}\right]
$$

Consequently, the analytical expression of information matrix is

$$
\mathbf{A}^{T} \mathbf{A}=F^{2}\left[\begin{array}{ll}
M_{11} & M_{12} \\
M_{21} & M_{22}
\end{array}\right]
$$

where

$$
\begin{aligned}
& M_{11}=\left\|\mathbf{J}_{1}\right\|^{4} \cos ^{2}\left(\varphi_{1}\right) \\
& M_{12}=M_{21}=\left\|\mathbf{J}_{1}\right\|^{2}\left\|\mathbf{J}_{2}\right\|^{2} \cos \left(\varphi_{0}\right) \cos \left(\varphi_{1}\right) \cos \left(\varphi_{2}\right) \\
& M_{22}=\left\|\mathbf{J}_{2}\right\|^{4} \cos ^{2}\left(\varphi_{2}\right)
\end{aligned}
$$

The obtained expression is quite suitable for formulating the optimization functions for both existing and proposed criteria. Let us address this in the following sections.

\section{Evaluation of Existing Optimality Criteria}

For illustrative purposes, the comparison study has been carried out for 2-link manipulator with the following parame- 
Table 2. Comparison of numerical optimization results for existing optimality criteria $\left(l_{1}=0.6 \mathrm{~m}, l_{2}=0.4 \mathrm{~m}, F=1 \mathrm{~N}\right)$

\begin{tabular}{ccccc}
\hline \hline \multirow{5}{*}{ Criterion } & \multicolumn{3}{c}{ Optimal Pose $[\mathrm{deg}]$} & \multicolumn{3}{c}{ Identification Accuracy $\left[\mathrm{rad}^{2} / \mathrm{N}^{2}\right]$} \\
\cline { 2 - 5 } & $q_{1}$ & $q_{2}$ & $\mathrm{E}\left[\delta k_{1}{ }^{T} \delta k_{1}\right]$ & $\mathrm{E}\left[\delta k_{2}{ }^{T} \delta k_{2}\right]$ \\
T-optimality & 0 & 0 & $\inf$ & $\inf$ \\
A-optimality & -83.34 & 98.79 & 13.7315 & 53.4130 \\
D-optimality & -43.72 & 62.93 & 5.3208 & 113.3093 \\
MDC & 78.16 & 112.17 & 44.2991 & 44.2991 \\
\multicolumn{5}{c}{ SVD-based Observability Indices } \\
$O_{1}$ & -43.72 & 62.93 & 5.3208 & 113.3093 \\
$O_{2}, O_{4}$ & 86.85 & 131.80 & 64.06 & 64.06 \\
$O_{3}$ & 85.40 & 112.57 & 29.4947 & 47.2227 \\
\hline \hline
\end{tabular}

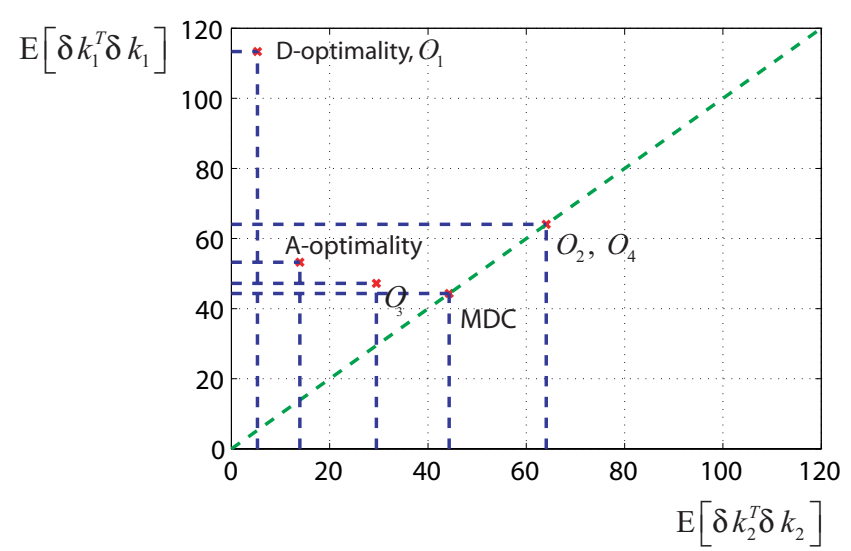

Figure 3. Identification accuracy of elasto-static parameters using different optimization criteria

ters: the link lengths $l_{1}=0.6 \mathrm{~m}, l_{2}=0.4 \mathrm{~m}$ and force magnitude $F=1 \mathrm{~N}$. The numerical results for the case of one calibration experiment for different optimization objectives are presented in Table 2. They include corresponding optimal measurement poses and the identification accuracy of the elasto-static parameters. For comparison, the latter are also illustrated in Fig. 3.

The results show that the solution for the T-optimality corresponds to a kinematically singular configuration with an infinite covariance matrix, which is not suitable for practical applications. Besides, solutions for $\mathrm{O}_{2}$ and $\mathrm{O}_{4}$ are dominated by the solutions for A-optimality, $\mathrm{O}_{3}$ and MDC (maximal diagonal covariance). So, they cannot be treated as the optimal ones for elasto-static calibration. Further, the MDC, $\mathrm{O}_{3}, \mathrm{~A}-$ and Doptimality principles provide a set of so-called Pareto optimal solutions, which do not dominate each other. However, they can be compared using a single scalar performance measure proposed in

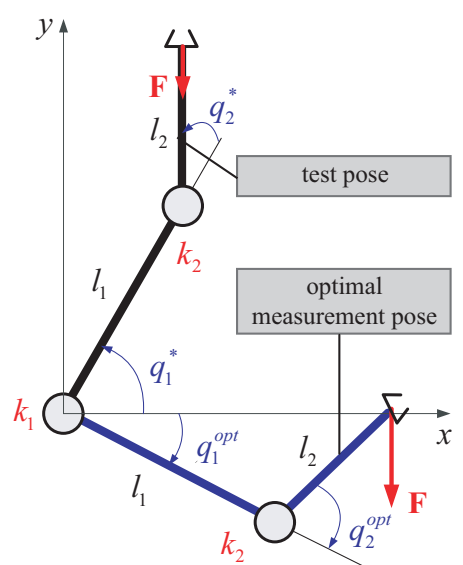

Figure 4. Test pose and corresponding optimal measurement pose of 2-link manipulator

this paper (the s.t.d. of the position errors after compensation of the elasto-static deflections). Using this approach, the common difficulty (defining the objective function) of multi-objective optimization can be overcome. As a result, the possibility to obtain a unique optimal solution is rather high, and the quality of associated calibration plans (measurement poses) is estimated by a physically clear performance measure: the degree of compliance errors compensation. So, let us focus on the evaluation of the proposed criterion and its potential advantages comparing to the existing experimental design approaches.

\section{Evaluation of the Proposed Criterion}

Since the considered 2-link manipulator has two output variables $x$ and $y$ to be measured, and two parameters $k_{1}$ and $k_{2}$ to be identified, it is possible to calibrate it with either one or several measurement poses. Let us start from calibration with a single experiment and further address calibration with several experiments.

Simulations for the case of a single experiment have been carried out for the test pose with the joint angles $q_{1}^{*}=\pi / 3$, $q_{2}^{*}=\pi / 6$. It should be mentioned that the variables $q_{1}$ and $\varphi$ are dependent. So, the force direction can be fixed for the test pose and measurement pose. In this case $\varphi=-\pi / 2$ is used. The same numerical values for the manipulator link lengths are used as in previous subsection. The test pose and the optimal measurement pose $\left\{q_{1}^{o p t}, q_{2}^{o p t}\right\}$ obtained using the proposed criterion are presented in Fig. 4. As follows from this figure, they are located quite far from each other in the workspace. Such test pose is selected for two reasons: For the given test pose, the quality of different measurement poses used in the calibration experiments are illustrated in Fig. 5, among which the obtained optimal solutions ensure five times more accurate compensation of the compliance deflections. This improvement of the calibration accuracy is defined by a scalar factor $\chi$, which has the following 


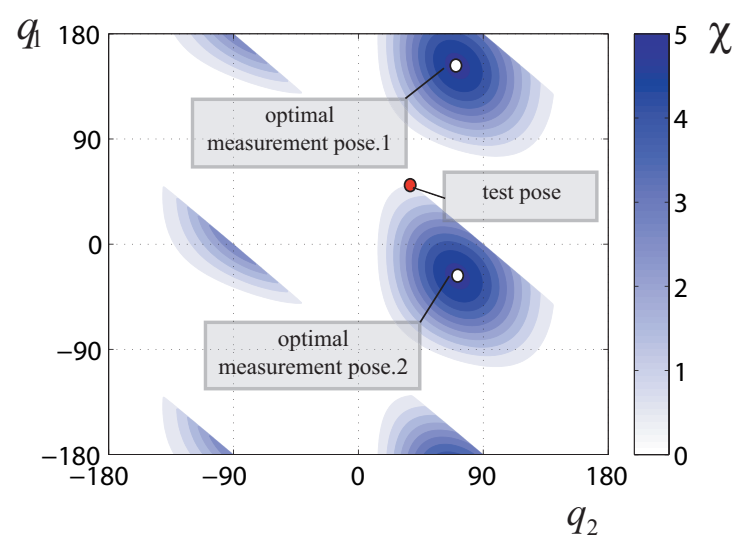

Figure 5. Accuracy improvement for different measurement poses

form

$$
\chi=\frac{O_{t}^{*}}{O_{t}}
$$

The higher the $\chi$ is, the more accurate the compensation is.

To demonstrate advantages of the proposed performance measure comparing to the existing ones, the expression (16) is used to evaluate the quality of the optimal calibration plans, which are associated with the Pareto solution obtained in the previous subsection. The corresponding values of $\chi$ are given in Table 3. Here, $\delta \tilde{k}_{1}$ and $\delta \tilde{k}_{2}$ refer to the mean square errors in identification of elasto-static parameters. For the presented manipulator, it is evident that the precision of $k_{1}$ has higher influence on the end-effector positioning accuracy than $k_{2}$. For this reason, the criteria MDC and $O_{3}$, which aim at balancing/eliminating the differences between $\delta \tilde{k}_{1}$ and $\delta \tilde{k}_{2}$, have decreased the desired accuracy of the error compensation. On the other hand, using the A- and D-optimality $\left(O_{1}\right)$ principles, yield, increasing of the accuracy by the factor of 1.7 and 4.5 respectively, but the proposed criterion ensures the best compliance error compensation.

The most important issue derived from the simulation results can be summarized in the following remark:

Remark 4 The maximal level of accuracy improvement varies with the test pose.

In order to make the results more representative, the inverse of $\chi$ is used to ensure the resulting values are bounded within $[0,1]$. So, the smaller value of $\frac{1}{\chi}$ is, the higher the maximal accuracy improvement is. It is shown in Fig. 6 that if the test pose is in the dark areas, the benefits of test-pose approach are minimum. In this case, it is possible to use the test pose as the calibration configuration. But, in most cases, the improvements are essential, the average value of it is 7.08 . The most significant change occurs when the test pose is in the white areas, the degree of com-

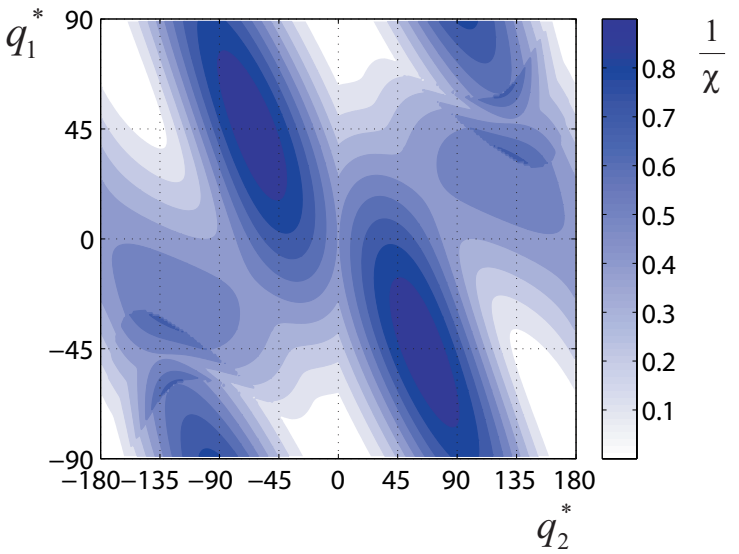

Figure 6. Maximum accuracy improvement for different test poses

Table 3. Comparison of accuracy improvement using different optimality criteria

\begin{tabular}{ccccc}
\hline \hline Criterion & $\chi$ & $\delta \tilde{k}_{1}[\mathrm{rad} / \mathrm{N}]$ & $\delta \tilde{k}_{2}[\mathrm{rad} / \mathrm{N}]$ & $O_{t}$ \\
\hline$O_{t}^{*}$ & 1 & 11.1111 & $\inf$ & 2 \\
A-optimality & 1.7041 & 3.7056 & 7.3084 & 1.1736 \\
D-optimality $\left(O_{1}\right)$ & 4.4632 & 2.3067 & 10.6447 & 0.4481 \\
MDC & 0.5361 & 6.6558 & 6.6558 & 3.7305 \\
$O_{3}$ & 0.8052 & 5.4309 & 6.8719 & 2.4838 \\
$O_{t}$ & 5.1694 & 2.1434 & 12.5104 & 0.3869 \\
\hline \hline
\end{tabular}

pensation accuracy is raised maximumly, by the factor of 10 .

For the considered manipulator, its optimal measurement pose for calibration with respect to a given test pose can be selected using the maps presented in Fig. 7. Furthermore, taking into account different link length ratio $b=l_{2} / l_{1}$, the maps for selecting optimal measurement poses for $b$ equal to $0.2,0.5$ and 0.9 are obtained and illustrated in Figs. 8-10. Thus, using the developed technique, for a 2-link manipulator with a given test pose and known link length ratio, it is possible to obtain the optimal measurement pose for calibration experiments and the corresponding level of accuracy improvement.

For the case of several calibration experiments, the simulation results (for two and three measurement poses) are presented in Tables 4 and 5. For comparison purposes, four different calibration plans are used:

(i) obtained by repeating the same measurement pose for several experiments.

(ii) obtained by optimization of $O_{t}$ with respect to $q_{1}$.

(iii) obtained by optimization of $O_{t}$ with respect to $q_{2}$.

(iv) obtained by optimization of $O_{t}$ with respect to $q_{1}$ and $q_{2}$. 

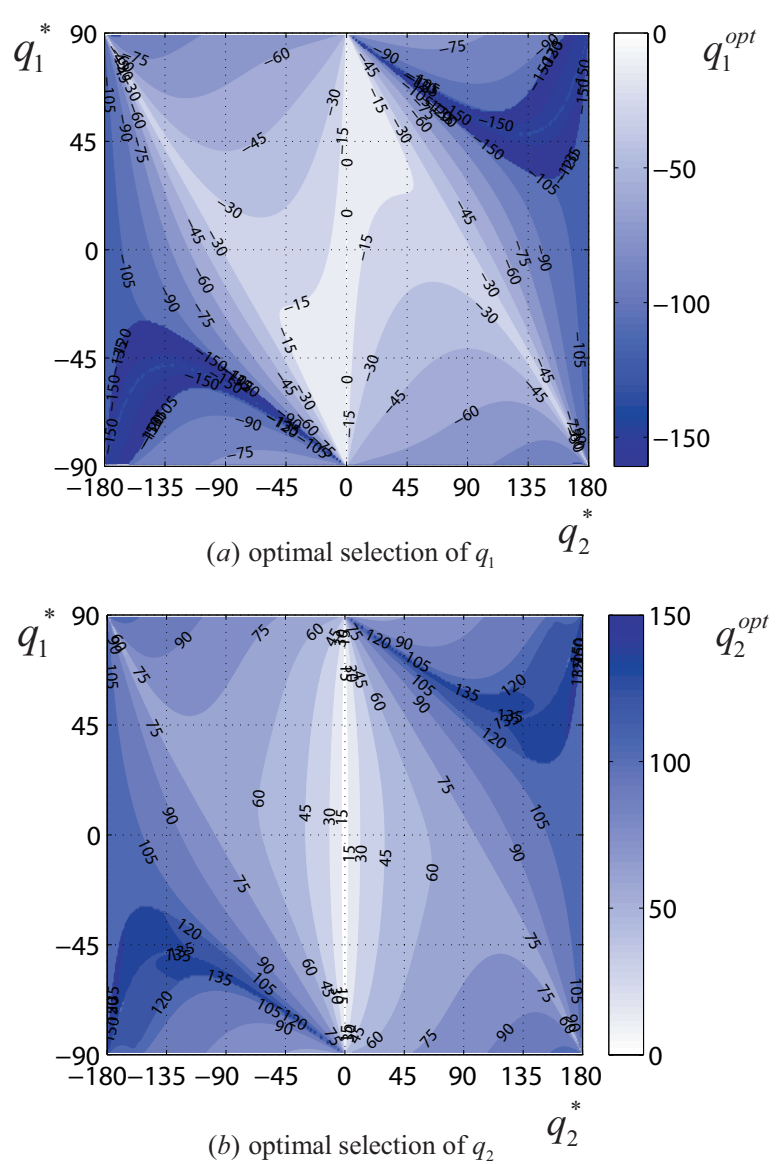

Figure 7. Maps for optimal measurement pose selection: $l_{2} / l_{1}=0.67$

The simplest way is to repeat several times the calibration using the same measurement pose $\left\{q_{1}^{o p t}, q_{2}^{o p t}\right\}$, which is obtained as the optimal one for a single calibration experiment (case (i)). This must obviously lead to the improvement of accuracy in accordance with expression (17). However, it is evident that the best results can be obtained using the global optimization (case (iv)), but this approach is more computational consuming.

Table 4 includes comparison of all above mentioned cases for two calibration experiments. The results show that cases (i) and (ii) produce almost the same values for the objective function, and the difference in identification accuracy is not significant. This indicates that changing in the variable $q_{1}$ has no contribution to the accuracy improvement. On the other hand, case (iii) (optimization with respect to $q_{2}$ ) has more influence. The identification error in $k_{2}$ is reduced significantly (almost twice), but the improvement in the compliance errors compensation is still negligible (less than 1\%). For case (iv), in spite of the error in $k_{2}$ is higher than in case (iii), the degree of error compensation is increased by $8 \%$ with regard to all other cases. So, the precision of $k_{1}$ is more important than $k_{2}$. It should be mentioned that,
Table 4. Numerical optimization results with different calibration plans: case of two experiments

\begin{tabular}{cccccc}
\hline \hline Variables & $O_{t}$ & $\begin{array}{c}q_{1} \\
{[\mathrm{deg}]}\end{array}$ & $\begin{array}{c}q_{2} \\
{[\mathrm{deg}]}\end{array}$ & $\begin{array}{c}\mathrm{E}\left[\delta k_{1}{ }^{T} \delta k_{1}\right] \\
{\left[\mathrm{rad}^{2} / \mathrm{N}^{2}\right]}\end{array}$ & $\begin{array}{c}\mathrm{E}\left[\delta k_{2}{ }^{T} \delta k_{2}\right] \\
{\left[\mathrm{rad}^{2} / \mathrm{N}^{2}\right]}\end{array}$ \\
\hline$q_{1}^{\text {opt }}, q_{2}^{\text {opt }}$ & 0.1934 & -27.50 & 71.34 & 2.2971 & 78.2531 \\
& & -27.50 & 71.34 & & \\
$q_{1}, q_{2}^{\text {opt }}$ & 0.1934 & -21.97 & 71.34 & 2.2971 & 78.8330 \\
& & -32.15 & 71.34 & & \\
$q_{1}^{\text {opt }}, q_{2}$ & 0.1920 & -27.50 & 41.22 & 2.2803 & 34.6372 \\
& & -27.50 & -145.05 & & \\
$q_{1}, q_{2}$ & 0.1640 & -131.81 & 131.81 & 1.9474 & 36.5317 \\
& & 180.00 & -29.12 & & \\
\hline \hline
\end{tabular}

Table 5. Numerical optimization results with different calibration plans: case of three experiments

\begin{tabular}{cccccc}
\hline \hline Variables & $O_{t}$ & $\begin{array}{c}q_{1} \\
{[\mathrm{deg}]}\end{array}$ & $\begin{array}{c}q_{2} \\
{[\mathrm{deg}]}\end{array}$ & $\begin{array}{c}\mathrm{E}\left[\delta k_{1}{ }^{T} \delta k_{1}\right] \\
{\left[\mathrm{rad}^{2} / \mathrm{N}^{2}\right]}\end{array}$ & $\begin{array}{c}\mathrm{E}\left[\delta k_{2}{ }^{T} \delta k_{2}\right] \\
{\left[\mathrm{rad}^{2} / \mathrm{N}^{2}\right]}\end{array}$ \\
\hline$q_{1}^{\text {opt }}, q_{2}^{\text {opt }}$ & 0.1290 & -27.50 & 71.34 & 1.5314 & 52.1687 \\
& & -27.50 & 71.34 & & \\
& & -27.50 & 71.34 & & \\
$q_{1}, q_{2}^{\text {opt }}$ & 0.1290 & 0.03 & 71.34 & 1.5314 & 59.9693 \\
& & -13.14 & 71.34 & & \\
& & -45.51 & 71.34 & & \\
$q_{1}^{\text {opt }}, q_{2}$ & 0.1192 & -27.50 & 55.69 & 1.4157 & 26.9694 \\
& & -27.50 & 55.69 & & \\
& & -27.50 & -146.41 & & \\
$q_{1}, q_{2}$ & 0.1039 & 0 & 46.10 & 1.2340 & \\
& & -131.81 & 131.81 & & \\
& & -180.00 & -46.10 & & \\
\hline \hline
\end{tabular}

comparing to one calibration experiment, the global optimization of calibration plan allows increasing the efficiency of error compensation by the factor of 1.5 (instead of the usual 1.4).

Similar results were obtained for the case of three calibration experiments and presented in Table 5. Here, the global optimization permits increasing the degree of error compensation by $10 \%$ with regard to other cases, and by the factor of 1.9 (instead of the usual 1.7) comparing to calibration with one experiment. In this case, the mean square errors in the identified parameters $k_{1}$ and $k_{2}$ can be reduced to $1.11(\mathrm{rad} / \mathrm{N})$ and $5.68(\mathrm{rad} / \mathrm{N}) \mathrm{respec}-$ tively. Hence, the proposed test-pose approach allows increasing the identification accuracy as well as the degree of error compen- 


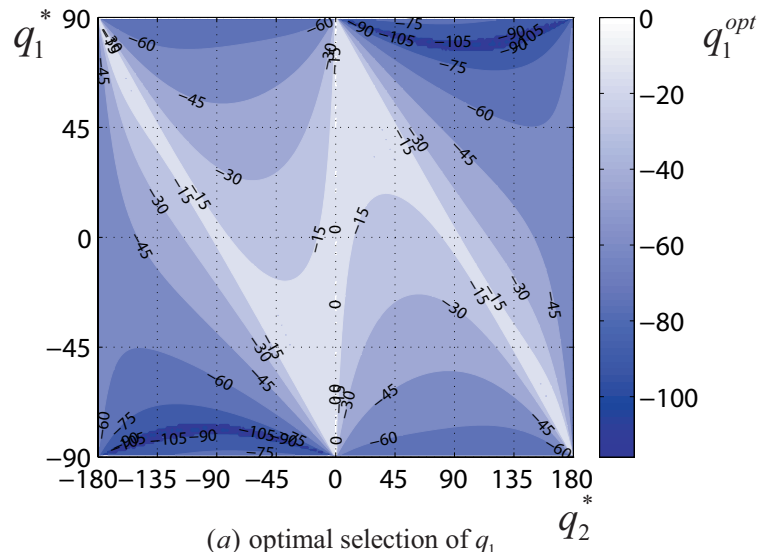

(a) optimal selection of $q$

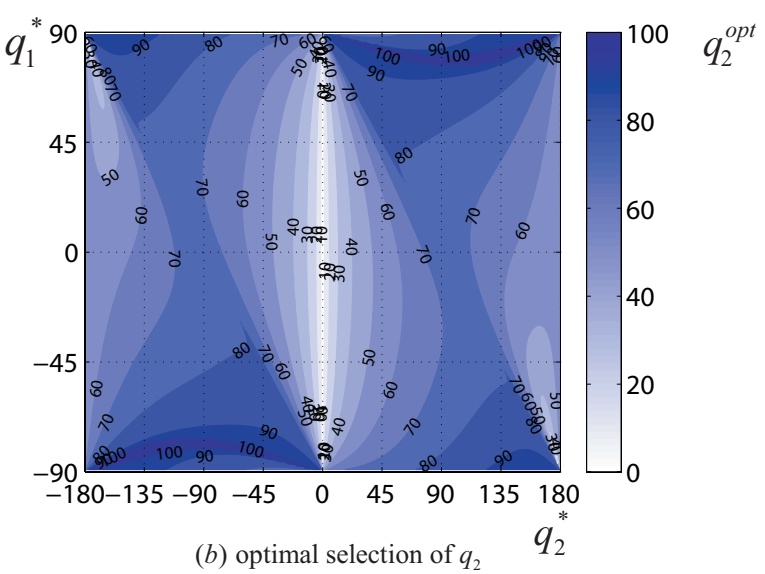

Figure 8. Maps for optimal measurement pose selection for $b=0.2$

sation significantly.

\section{CONCLUSIONS}

In this paper, a new criterion for selection of measurement poses for elasto-static calibration is proposed. It is based on the concept of the manipulator test-pose, and evaluates the degree of compliance errors compensation. The proposed criterion allows essentially improving the identification accuracy via proper selection of the manipulator measurement poses that are used in calibration. In contrast to the existing criteria, it has a clear physical meaning that is directly related to the robot accuracy, and permits to combine multiple objectives with different units into a single scalar expression. It was proved that this approach is an extension of the conventional A-optimality principle known from the design of experiments theory, and the proposed criterion can be expressed as the weighted trace of the covariance matrix, where the weighting coefficients are derived using the test pose.

The advantages of the proposed criterion and the comparison study with conventional ones were illustrated via simulation study that deals with a 2-link planar manipulator. For this manip-
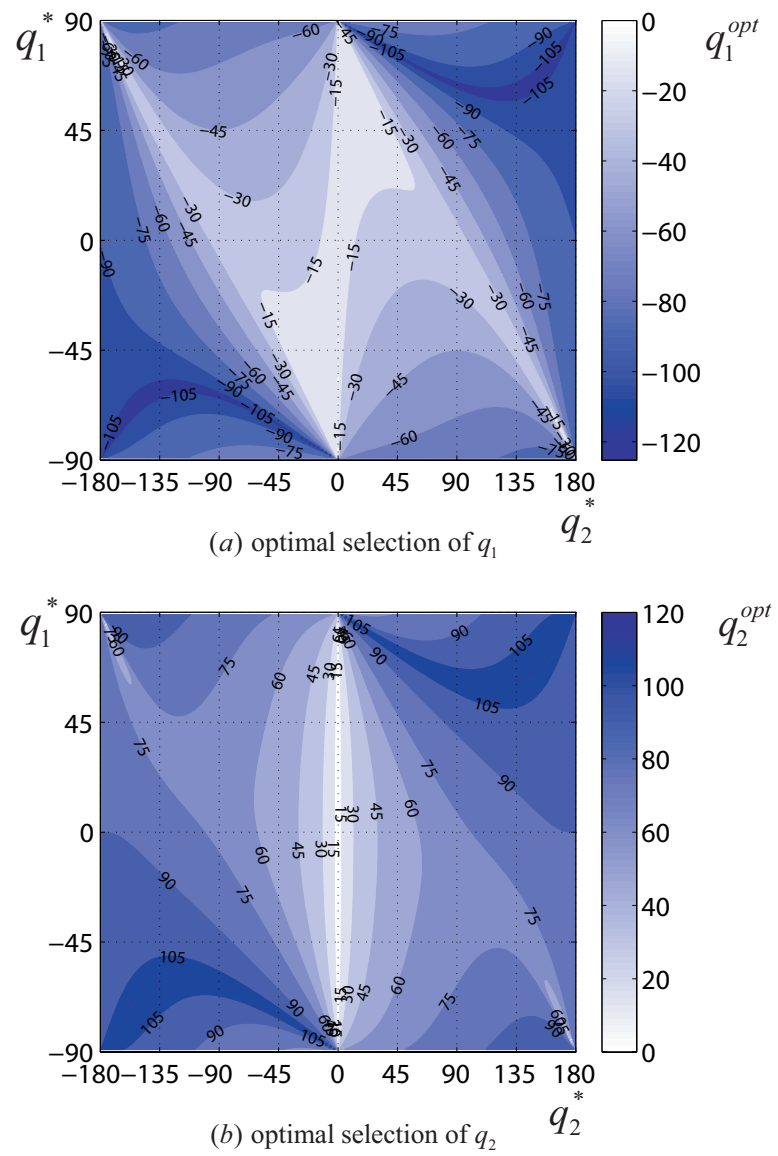

Figure 9. Maps for optimal measurement pose selection for $b=0.5$

ulator, the maps for selecting optimal measurement poses with respect to the given test pose were proposed for different link length ratio.

\section{ACKNOWLEDGMENTS}

The authors would like to acknowledge the financial support of the French "Agence Nationale de la Recherche"(Project ANR2010-SEGI-003-02-COROUSSO) and the Region "Pays de la Loire", France.

\section{REFERENCES}

[1] Meggiolaro, M. A., Dubowsky, S., Mavroidis,C., 2005, "Geometric and Elastic Error Calibration of a High Accuracy Patient Positioning System", Mechanism and Machine Theory, 40, pp. 415-427.

[2] Elatta, A. Y., Li, P., Fan, L., Yu, D., and Lou, F., 2004, “An Overview of Robot Calibration", Information Technology Journal, 3, pp. 74-78.

[3] Hollerbach, J., M., 1989, "A survey of kinematic calibration", The Robotics Review, 1, pp. 207-242.

[4] Khalil, W., Besnard, S., 2002, "Geometric calibration of 


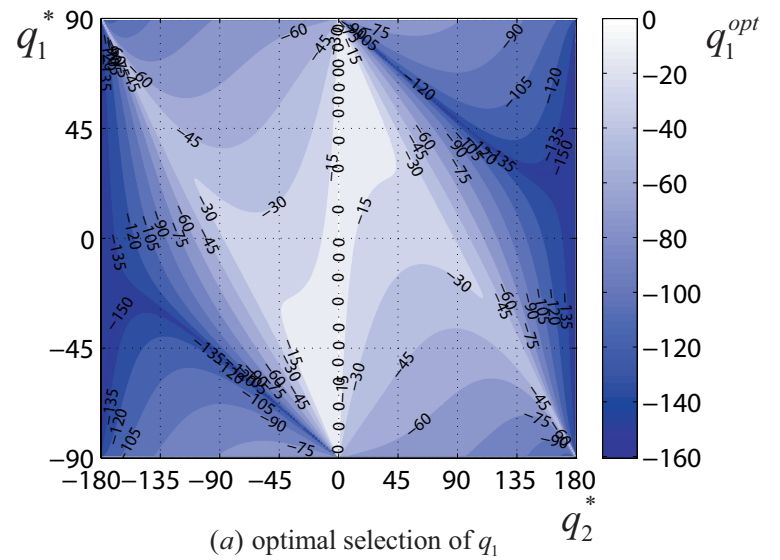

(a) optimal selection of $q_{1}$

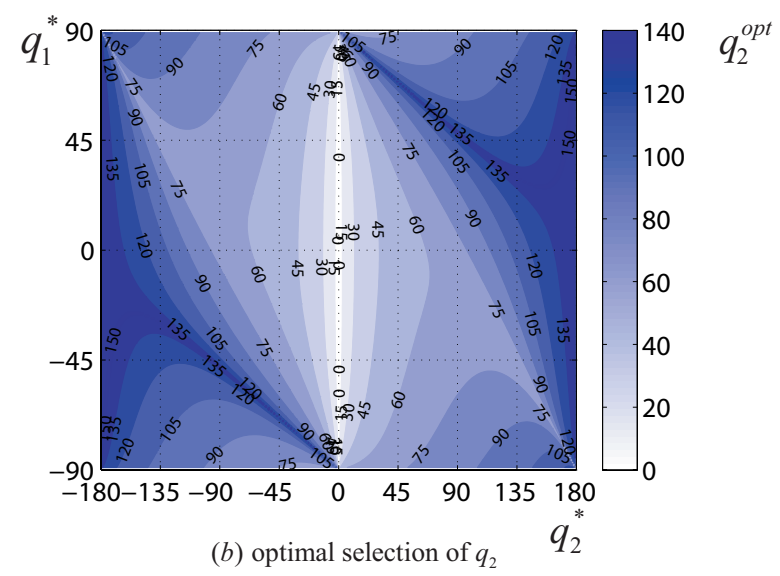

Figure 10. Maps for optimal measurement pose selection for $b=0.9$

robots with flexible joints and links", Journal of Intelligent and Robotic Systems, 34, pp. 357-379.

[5] Daney, D., Andreff, N., Chabert, G., Papegay, Y., 2006, "Interval Method for Calibration of Parallel Robots: Visionbased Experiments", Mechanism and Machine Theory, 41, pp. 929-944.

[6] Hollerbach, J., M., Khalil, W., Gautier, M., 2008, "Handbook of Robotics", Springer, Chap. Model identification, pp. 321-344.

[7] Gong, C., Yuan J., and Ni,J., 2000, "Nongeometric Error Identification and Compensation for Robotic System by Inverse Calibration", International Journal of Machine Tools and Manufacture, 40, pp. 2119-2139.

[8] Bogdan, I., C., Abba, G., 2009, "Identification of the Servomechanism Used for Micro-displacement", IROS, IEEE/RSJ International Conference, pp. 1986-1991.

[9] Daney, D., 2002, "Optimal Measurement Configurations for Gough Platform Calibration", Robotics and Automation, ICRA/ IEEE International Conference, 1, pp. 147-152.

[10] Daney, D., Papegay, Y., Madeline, B., 2005, "Choosing Measurement Poses for Robot Calibration with the Local
Convergence Method and Tabu Search", The International Journal of Robotics Research, 24(6), pp. 501-518.

[11] Klimchik, A., Wu, Y., Caro, S., Pashkevich, A., 2011, "Design of Experiments for Calibration of Planar Anthropomorphic Manipulators", Advanced Intelligent Mechatronics (AIM), IEEE/ASME International Conference, pp. 576581.

[12] Zhuang, H., Wang, K., Roth, Z. S., 1994, "Optimal Selection of Measurement Configurations for Robot Calibration Using Simulated Annealing", Robotics and Automation, IEEE International Conference, 1, pp. 393-398.

[13] Driels1, M., R., Pathre, U., S., 1990, "Significance of Observation Strategy On the Design of Robot Calibration Experiments", Journal of Robotic Systems, 7(2), pp. 197-223.

[14] Mitchell, T., J., 1974, "An Algorithm for the Construction of D-optimality Experimental Designs", Techometrics, 16(2), pp. 203-210.

[15] Yu, S. and Hollerbach, J., M., 2008, "Observability Index Selection for Robot Calibration", Robotics and Automation, ICRA, IEEE International Conference, pp. 831-836.

[16] Fiefer, J. and Wolfowitz, J., 1959, "Optimum Designs in Regression Problem", Annals of Mathematical Statistics, 23, pp.271-294.

[17] Nahvi, A. and Hollerbach, J., M., 1996, "The Noise Amplification Index for Optimal Pose Selection in Robot Calibration", Robotics and Automation, 1, pp. 647-654.

[18] Borm, J., H. and Menq, C., H., 1991, "Determination of Optimal Measurement Configurations for Robot Calibration Based on Observability Measure", Journal of Robotic Systems, 10(1), pp.51-63.

[19] Khalil, W., Gautier, M., Enguehard, Ch., 1991, "Identifiable parameters and optimum configurations for robots calibration", Robotica, 9, pp. 63-70.

[20] Zhuang, H., Wu, J., Huang, W., 1996, "Optimal Planning of Robot Calibration Experiments by Genetic Algorithms", Robotics and Automation, IEEE, 2, pp. 981-986

[21] Pashkevich, A., Klimchik, A., Chablat, D., 2011, "Enhanced Stiffness Modeling of Manipulators with Passive Joints", Mechanism and Machine Theory, 46, pp. 662-679.

[22] Cox, D., R., 1957, "Planning of Experiments.", Oxford, England.

[23] Allen, D., M., 1971, "Mean Square Error of Prediction as a Criterion for Selecting Variables", Technometrics, 13(3), pp. 469-475. 